\title{
THE GRAPH OF AN ABSTRACT POLYTOPE*
}

\author{
Katta G. MURTY \\ University of Michigan, Ann Arbor, Michigan, U.S.A.
}

Received 24 May 1972

Revised manuscript received 12 February 1973

\begin{abstract}
Recently a generalization of simple convex polytopes to combinatorial entities known as abstract polytopes has been proposed. The graph of an abstract polytope of dimension $d$ is a regular connected graph of degree $d$. Given a connected regular graph $\Gamma$ of degree $d$, it is interesting to find out whether it is the graph of some abstract polytope $P$. We obtain necessary and sufficient conditions for this, in terms of the existence of a class of simple cycles in $\Gamma$ satisfying certain properties. The main result in this paper is that if a pair of simple convex polytopes or abstract polytopes have the same two-dimensional skeleton, then they are isomorphic. Every twodimensional face of a simple convex polytope or an abstract polytope is a simple cycle in its graph. Given the graph of a simple convex polytope or an abstract polytope and the simple cycles in this graph corresponding to all its two-dimensional faces, then we show how to construct all its remaining faces. Given a regular connected graph $\Gamma$ and a class of simple cyles $D$ in it, we provide necessary and sufficient conditions under which $D$ is the class of two-dimensional faces of some abstract polytope which has $\Gamma$ as its graph.
\end{abstract}

\section{Introduction}

\subsection{Let $K$ be the set of all feasible solutions of}

$$
A x=b, \quad x \geqq 0,
$$

where $A$ is a matrix of order $m \times n$ and rank $m$. Suppose $K$ is nonempty and bounded. Also assume that the column vector $b$ is nondegenerate in (1), i.e., that it does not lie in any subspace generated by $m-1$ or less column vectors of $A$. Under these assumptions, $K$ is a simple convex polytope of dimension $d=n-m$ and $\tilde{x} \in K$ is an extreme point iff exactly $m$ of the $\widetilde{x}_{j}$ are positive and the remaining $n-m$ are zero.

* This research has been partially supported by the ISDOS Research Project at the Department of Industrial and Operations Engineering, and by the National Science Foundation under Grant No. GK-27872 with the University of Michigan. 
Let $S=\{1, \ldots, n\}$. Corresponding to each extreme point $\widetilde{x}$ of $K$, define

$$
V(\widetilde{x})=\left\{j: j \text { such that } \tilde{x}_{j}=0\right\} .
$$

Each $V(\widetilde{x})$ is a subset of $S$ of cardinality $d=n-m$ and will be called a vertex. Let $C$ be the class of all the vertices. Let $P(K)$ denote the pair $(S, C)$. Clearly $S^{*}=\cup_{V \in C} V$ may not be all of $S$.

In analogy with the definition of adjacency of extreme points on $K$, we define that two vertices $V_{1}$ and $V_{2}$ in $P(K)$ are adjacent iff $V_{1} \cap V_{2}$ has cardinality equal to the cardinality of any vertex minus one. From the theory of linear programming, we know that $P(K)$ satisfies the following:

1.1.1. The class $C$ of vertices is nonempty.

1.1.2. Every vertex is a subset of $S$ of the same cardinality.

1.1.3. If $E$ is a subset of $S$ of cardinality equal to the cardinality of a vertex minus one, then the number of vertices which contain $E$ as a subset is either 0 or 2 .

1.1.4. If $V_{0}$ and $V_{*}$ are a pair of vertices, then there exists a sequence of vertices $V_{0}, V_{1}, V_{2}, \ldots, V_{t}, V_{*}$, known as an adjacency path or edge path between $V_{0}$ and $V_{*}$, such that every pair of consecutive vertices in the sequence are adjacent and every vertex in the sequence contains $V_{0} \cap V_{*}$ as a subset.

1.2. The pair $P(K)=(S, C)$ is known as an abstract polytope corresponding to the simple convex polytope $K . P(K)$ captures the essential adjacency structure between the extreme points of $K$.

In general, any pair $(S, C)$ satisfying 1.1.1-1.1.4 defines an abstract polytope. In analogy to simple convex polytopes, we define the dimen sion of the abstract polytope $(S, C)$ to be the cardinality of any vertex in $C$.

1.3. Faces. A nonempty face of the convex polytope $K$ is a nonempty set of feasible solutions of (1) after setting some of the variables $x_{j}$ to zero. Analogously, let $P=(S, C)$ be an abstract polytope and $F \subset S$. Let $C_{F}=\{V \backslash F: V \in C, V \supseteq F\}$, where $\backslash$ indicates set-theoretic difference. If $C_{F}$ is nonempty, it is clear that $\left(S, C_{F}\right)$ is itself an abstract polytope, and we call it the nonempty face of $P$ generated by the subset $F$ of $S$. The dimension of this face is equal to the dimension of $P$ minus the cardinality of $F$. Two faces $\left(S, C_{F_{1}}\right)$ and $\left(S, C_{F_{2}}\right)$ are distinct if $F_{1} \neq F_{2}$. 
Clearly a nonempty face of dimension 0 of an abstract polytope $P=(S, C)$ is generated by a subset of $S$ which is itself a vertex, and hence it contains only that vertex.

A nonempty face of dimension $!$ is generated by a subset of $S$ of cardinality equal to the dimension of $P$ minus one, and it contains exactly two adjacent vertices of $P$. It will be called an edge of $P$.

1.4. Graph. The graph of an abstract polytope $P=(S, C)$ is obtained by representing each vertex in $C$ by a vertex of the graph and joining a pair of vertices by an edge of the graph iff these vertices are adjacent on $P$. The graph is just the one-dimensional skeleton of $P$, and is a connected regular graph of degree equal to the dimension of $P$.

1.5. The axioms defining abstract polytopes were first outlined by Dantzig and discussed in [3]. References [1-3, 6] contain several results on abstract polytopes analogous to known results on convex polytopes.

1.6. Suppose that $\Gamma$ is a given connected regular graph of degree $d$. If we find a finite set of symbols $S=\{1,2, \ldots, n\}$ and a labelling of the vertices of $\Gamma$ by subsets of $S$ of cardinality $d$ such that when $C$ is the class of labels on the vertices of $\Gamma,(S, C)$ is an abstract polytope and two vertices of $\Gamma$ are connected by an edge of $\Gamma$ iff the labels on these vertices represent a pair of adjacent vertices of $(S, C)$, then we say that the vertices of $\Gamma$ have been labelled to be the vertices of an abstract polytope $(S, C)$. We obtain necessary and sufficient conditions for the existence of such a labelling.

We also obtain the result that two abstract polytopes which have the same two-dimensional skeleton are isomorphic. Since the class of abstract polytopes includes all simple convex polytopes, this result also holds for simple convex polytopes. For other related results on the $k$ skeletons of convex polytopes, see [5, ch. 12].

Finally, the connections between abstract polytopes and simplicial complexes in combinatorial topology are easy to see. In the $P(K)=(S, C)$ derived from the simple convex polytope $K$, the vertices in $C$ are the $d-1$ simplices of a-simplicial complex which is isomorphic to the boundary complex of the simplicial polytope dual to $K$. See [4, chs. 4,5$]$. 


\section{The results}

2.1. Lemma. The graph of an abstract polytope $P$ of dimension 2 is a simple cycle.

Proof. By definition, the graph of $P$ is a connected graph of degree 2 and is therefore a simple cycle.

2.2. Lemma. Let $\Gamma$ be the graph of an abstract polytope $P=(S, C)$ of dimension $d \geqq 3$. Let $D=\left\{c_{1}, \ldots, c_{T}\right\}$ be the class of all the simple cycles in $\Gamma$ determined by the two-dimenisional faces of $P$. Then:

(i) If a pair of simple cycles in D contain two common vertices, then they must be adjacent and these cycles have no other vertex in common. Thus, a pair of simple cycles in 0 contain either exactly one common edge or no common edges at all.

(ii) Each edge of $\Gamma$ occurs in exactly $\mathrm{d}-1$ simple cycles in $D$; or, equivalently, for every pair of edges of $\Gamma$ with a common vertex, there is a unique simple cycle in Dcontaining both of them.

Proof. (i) Let $c_{1}, c_{2}$ be the simple cycles in $D$ which are the twodimensional faces generated by $F_{1} \subset S$, and $F_{2} \subset S$, respectively, $F_{1} \neq F_{2}$. If $V_{0}, V_{1}$ are two distinct vertices which are on both $c_{1}$ and $c_{2}$, then both $V_{0}, V_{1}$ must contain both $F_{1}, F_{2}$ as subsets. Since both $F_{1}$ and $F_{2}$ have cardinality $d-2$, this implies that $F_{1} \cup F_{2}=V_{0} \cap V_{1}$ has cardinality $d \cdots 1$ and hence $V_{0}, V_{1}$ must be adjacent. Since every common vertex of $c_{1}$ and $c_{2}$ must contain $F_{1} \cup F_{2}$ as a subset, 1.1.3, $V_{0}$ and $V_{1}$ are the only two common vertices of $c_{1}$ and $c_{2}$. This also implies that any pair of simple cycles in $D$ can have at most one common edge.

(ii) Let $e_{1}$ be an edge of $\Gamma$ which is the one-dimensional face of $P$ generated by $F_{3}$. Both the vertices on $e_{1}$ contain $F_{3}$ as a subset. Hence every subset of $F_{3}$ of cardinality $d-2$ generates a two-dimensional face of $P$. Obviously there are $d-1$ of these two-dimensional faces, and by (i) these are the only two-dimensional faces containing $e_{1}$.

$\Gamma$ is a regular graph of degree $d$. Let $V_{1}$ be a vertex on $e_{1}$, and let $e_{2}, \ldots, e_{d}$ be the other edges incident at $V_{1}$. By the above arguments, $e_{1}$ is contained among $d-1$ simple cycles in the class $D$ and the edge $e_{1}$ is the only common edge between any pair of these simple cycles. This implies that there is a unique simple cycle in $D$ passing through each pair of the edges $e_{1}, e_{j}$ for $2 \leqq j \leqq d$. 
2.3. Lemma. Let $\Gamma$ be the graph of an abstract polytope $P=(S, C)$ of dimension $d$. Let $e_{1}, \ldots, e_{r}$ be the edges of $\Gamma$ incident at a vertex of $\Gamma$. Then there is a unique $r$-dimensional face of $\Gamma$ containing all the edges $e_{1}, \ldots, e_{r}$.

Proof. Let $V_{0}=\{1, \ldots, d\}$ be the common vertex on $e_{1}, \ldots, e_{r}$. Each subset of $V_{0}$ of cardinality $d-1$ generates an edge containing $V_{0}$. Let $e_{i}$ be the edge generated by the subset $\{1, \ldots, i-1, i+1, \ldots, d\}$, for $i=1, \ldots, r$. Then clearly the $r$-dimensional face of $P$ generated by the subset $\{r+1, \ldots, d\}$ is the unique $r$-dimensional face of $P$ which contains all the edges $e_{1}, \ldots, e_{r}$.

2.4. We will now explore the properties of the class of simple cycles which are the two-dimensional faces of an abstract polytope. In particular, we will develop a procedure for generating the faces of all dimensions of an abstract polytope using only the class of its two-dimensional faces.

2.5. Theorem. Let $\Gamma$ be the graph of an abstract polytope $P$ of dimension $r$. Let $D$ be the class of simple cycles in $\Gamma$, which are the two-dimensional faces of $P$. Let $V_{0}$ be a vertex with edges $e_{1}, \ldots, e_{r}$ incident at it. If a subclass $\gamma$ of $D$ is obtained by the rules

(a) for every pair of edges $e_{i}, e_{j}, 1 \leqq i<j \leqq r$ the unique simple cycle in $D$ which contains both $e_{i}, e_{j}$ is in $\gamma$,

(b) any simple cycle in $D$ which contains two edges from the edges on the simple cycles which are already in $\gamma$ is itself in $\gamma$, then $\gamma=0$.

Proof. Let $V_{1}$ be the other vertex at the end of edge $e_{1}$ and suppose $e_{r+1}, \ldots, e_{2 r-1}$ are the other edges incident at $V_{1}$ (see fig. 1).

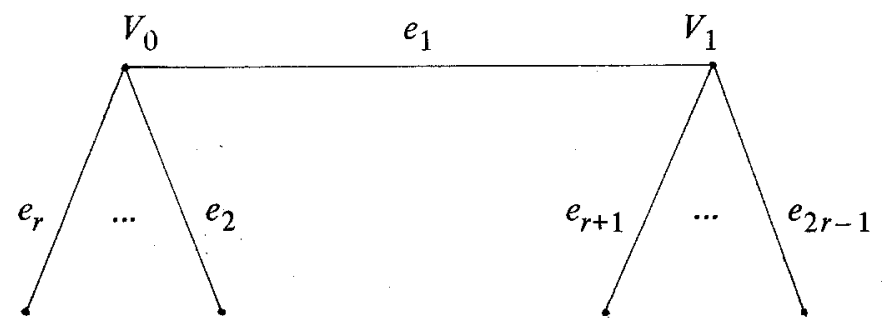

Fig. 1 . 
By Lemma 2.2, the simple cycles in $D$ through the edges $e_{1}, e_{i}$ for $i=2, \ldots, r$ must each contain one distinct edge among $e_{r+1}, \ldots, e_{2 r-1}$. Each one of these simple cycles is in $\gamma$. Hence the fact that $\gamma$ contains all the simple cycles in $D$ which pass through the vertex $V_{0}$ implies by (b) that $\gamma$ contains all the simple cycles in $D$ passing through each adjacent vertex of $V_{0}$. Since $\Gamma$ is connected, this argument applied repeatedly yields that $\gamma=D$.

2.6. Theorem. Let $\Gamma$ be the graph of an abstract polytope $P=(S, C)$ of dimension d. Let $D=\left\{c_{1}, \ldots, c_{T}\right\}$ be the class of simple cycles in $\Gamma$ which are all the two-dimensional faces of $P$. Let $e_{1}, \ldots, e_{r}$ be edges of $\Gamma$ with a common vertex $V_{0}$. Let $D\left\{e_{1}, \ldots, e_{r}\right\}$ be the subclass of $D$ obtained by the rules

(a) for every pair of edges $e_{i}, e_{j}, 1 \leqq i<j \leqq r$, the unique simple cycle in $D$ which contains both $e_{i}, e_{j}$ is in $D\left\{e_{1}, \ldots, e_{r}\right\}$,

(b) any simple cycle in $D$ which contains two edges from the edges on the simple cycles which are already in $D\left\{e_{1}, \ldots, e_{r}\right\}$ is itself in $D\left\{e_{1}, \ldots, e_{r}\right\}$,

then $D\left\{e_{1}, \ldots, e_{r}\right\}$ is the class of all the two-dimensional faces of the unique r-dimensional face of $P$ containing $e_{1}, \ldots, e_{r}$.

Proof. The fact that $\mathcal{D}\left\{e_{1}, \ldots, e_{r}\right\}$ constructed in the above manner contains all the simple cycles corresponding to the two-dimensional faces. of the unique $r$-dimensional face of $P$ containing $e_{1}, \ldots, e_{r}$ follows from Lemma 2.3 and Theorem 2.5.

Let $V_{0}=\{1,2, \ldots, d\}$. For $1 \leqq i \leqq r$, let $e_{i}$ be the edge of $P$ generated by the subset $\{1, \ldots, i-1, i+1, \ldots, d\}$ of $S$. Then the $r$-dimensional face of $P$ generated by the subset $\{r+1, \ldots, d\}$ is the unique $r$-dimensional face of $P$ which contains all the edges $e_{1}, \ldots, e_{r}$.

If $F_{1}$ and $F_{2}$ are two subsets of $S$ which generate edges of $P$, then from 1.3 it is clear that both these edges lie on a two-dimensional face of $P$ iff $F_{1} \cap F_{2}$ has cardinality $d-2$, and in this case all the vertices on this two-dimensional face contain $F_{1} \cap F_{2}$ as a subset and all the edges on this two-dimensional face are generated by subsets of $S$ wich contain $F_{1} \cap F_{2}$ as their subset .

Hence all the edges on the two-dimensional faces which are included in $\mathcal{D}\left\{e_{1}, \ldots, e_{r}\right\}$ in step (a) are generated by subsets of $S$ which contain $\{r+1, \ldots, d\}$ as a subset. Applying this argument repeatedly, from (b) it is clear that every edge contained on the simple cycles included in $D\left\{e_{1}, \ldots, e_{r}\right\}$ is generated by some subset of $S$ which contains 
$\{r+1, \ldots, d\}$ as its subset. Hence none of the simple cycles in $D\left\{e_{1}, \ldots, e_{r}\right\}$ contain an edge which does not lie in the $r$-dimensional face of $p$ generated by $\{r+1, \ldots, d\}$.

These things imply that $D\left\{e_{1}, \ldots, e_{r}\right\}$ is the set of all simple cycles corresponding to the two-dimensional faces of the unique $r$-dimensional face of $P$ containing $e_{1}, \ldots, e_{r}$.

The partial subgraph of $\Gamma$ consisting of the vertices and edges appearing among the simple cycles in $\mathcal{D}\left\{e_{1}, \ldots, e_{r}\right\}$ is the graph of the $r$-dimensional face of $P$ containing $e_{1}, \ldots, e_{r}$.

2.7. Procedure. The two-dimensional skeleton of an abstract polytope consists of its graph $\Gamma$ and the class $D$ of simple cycles in $\Gamma$ which are all the two-dimensional faces $c$. $P$. Theorem 2.6 provides an easy procedure for generating the faces of all dimensions of $P$ given only its twodimensional skeleton.

Pick a set of $r$ edges of $\Gamma$, say $e_{1}, \ldots, e_{r}$, containing a common vertex. Then $D\left\{e_{1}, \ldots, e_{r}\right\}$ obtained by using (a) and (b) of Theorem 2.6 determines an $r$-dimensional face of $P$. By picking all possible subsets of $r$ edges containing a common vertex, all $r$-dimensional faces of $P$ can be generated in this manner. However, in this list, each $r$-dimensional face of $P$ is likely to appear several times. Duplication can be avoided by picking each time a set of $r$ edges with a common vertex, so that all these $r$ edges together did not appear in any $r$-dimensional face generated so far.

By varying $r$ from 3 to the dimension of $P$, all the faces of $P$ can be generated.

2.8. Theorem. A pair of abstract polytopes which have the same 2-dimensional skeleton are isomorphic.

Proof. By Theorem 2.6, and 2.7, it is clear that if two abstract polytopes have the same two-dimensional skeleton, then they have the same facial structure. Hence they are isomorphic.

2.9. Corollary. For the class of simple convex polytopes, the two-dimensional skeleton is unambiguous.

Proof. Since each simple convex polytope is an abstract polytope, this result follows from Theorem 2.8. See [5, ch 12] for other related results on $k$-dimensional skeletons of convex polytopes. 
2.10. We will now use these results to obtain necessary and sufficient conditions for a regular connected graph to be the graph of an abstract polytope. These turn out to be very simple when the degree of the graph is 3 and we discuss this case now.

2.11. Theorem. A regular connected graph $\Gamma$ of degree 3 is the graph of an abstract polytope iff there exists in $\Gamma$ a class $D=\left\{c_{1}, \ldots, c_{T}\right\}$ of simple cycles satisfying properties (i), (ii) of Lemma 2.2 with d equal to 3 .

Proof. The necessity part of this theorem follows from Lemma 2.2. To prove the sufficiency, suppose the class $D=\left\{c_{1}, \ldots, c_{T}\right\}$ of simple cycles in $\Gamma$ satisfying (i), (ii) of Theorem 2.2 with $d$ equal to 3 is given. Associate the symbol $j$ with the simple cycle $c_{j}$ in $D$, and let $S=\{1, \ldots, T\}$. By (ii), each vertex in $\Gamma$ lies in exactly 3 simple cycles in $D$. Let the label on each vertex be the subset of 3 symbols associated with the three simple cycles in $D$ containing that vertex. Let $C$ be the class of all the labels on the vertices of $\Gamma$. We will now prove that $(S, C)$ is an abstract polytope of dimension 3 and that $\Gamma$ is its graph. Obviously the pair $(S, C)$ satisfies the axioms 1.1.1 and 1.1.2.

Let $E$ be any subset of $S$ of cardinality 2, say $E=\{1,2\}$, and suppose there is a vertex whose label contains $E$ as a subset, say $V_{0}=\{1,2,3\}$. Let $e_{1}, e_{2}, e_{3}$ be the edges incident at $V_{0}$. Suppose the symbols $1,2,3$ are associated with the simple cycles $c_{1}, c_{2}, c_{3}$, containing the pairs of edges $\left(e_{1}, e_{2}\right),\left(e_{2}, e_{3}\right),\left(e_{3}, e_{1}\right)$, respectively. Then $e_{2}$ is common to both the simple cycles $c_{1}$ and $c_{2}$ and by (i) the two vertices on $e_{2}$ are the only two vertices whose labels contain $E$ as a subset. Hence axiom 1.1.3 is satisfied and adjacent vertices in $\Gamma$ have labels which have two symbols in common.

Let $V_{0}$ and $V_{*}$ be the labels on two vertices. If $V_{0} \cap V_{*}$ has cardinality 2 , then by the above argument these vertices are adjacent on $\Gamma$. If $V_{0} \cap V_{*}$ has cardinality 1 , then both these vertices lie on a simple cycle in $D$, and this simple cycle contains a path between these two vertices along the edges of $\Gamma$ such that the label on every vertex along the path contains $V_{0} \cap V_{*}$ as a subset.

If $V_{0} \cap V_{*}$ is empty, since $\Gamma$ is connected, there is a path between these two vertices along the edges of $\Gamma$. Thus axiom 1.1.4 is also satisfied.

Hence the pair $(S, C)$ is an abstract polytope of dimension 3 and $\Gamma$ is its graph. 
2.12. When $\Gamma$ is a regular connected graph of degree $d>3$, Adler [2] has constructed an example to show that the mere fact of the existence of a class of simple cycles in $\Gamma$ satisfying (i), (ii) of Lemma 2.2 is not sufficient to conclude that $\Gamma$ is the graph of an abstract polytope. So when $d>3$ we have to impose some more conditions. For this we introduce the following notation:

Let $\Gamma$ be a regular connected graph of degree $d>3$. Let $D$ be a class of simple cycles in $\Gamma$ satisfying (i) and (ii) of Lemma 2.2. For any $r$ such that $2 \leqq r \leqq d-1$, let $e_{1}, \ldots, e_{r}$ be a set of $r$ edges of $\Gamma$ with a common vertex. Define the subclass $D\left\{e_{1}, \ldots, e_{r}\right\}$ of $D$ by (a), (b) of Theorem 2.6. There is a subset $D\left\{e_{1}, \ldots, e_{r}\right\}$ of $D$ for every set of distinct edges $\left\{e_{1}, \ldots, e_{r}\right\}$ of $\Gamma$ with a common vertex. Make a list of all these subsets. In this list each subset $D\left\{e_{1}, \ldots, e_{r}\right\}$ is likely to appear several times . Let $F_{r}$ denote the family of all the distinct subsets of the form $D\left\{e_{1}, \ldots, e_{r}\right\}$.

By this procedure we therefore get the families $F_{2}, F_{3}, \ldots, F_{d-1}$ of subsets of $D$. Obviously each subset in the family $F_{2}$ consists of just one simple cycle in $D$.

2.13. Theorem. If $\Gamma$ is a connected regular graph of degree $d>3$, it is the graph of an abstract polytope iff there exists in $\Gamma$ a class $D=\left\{\varepsilon_{1}\right.$, $\left.\ldots, c_{T}\right\}$ of simple cycles satisfying the following properties:

D satisfies properties (i) and (ii) of Lemma 2.2 .

The families $F_{2}, \ldots, F_{d-1}$ of subsets of $D$ generated as in 2.12 satisfy the following properties:

(iii) The partial subgraph of $\Gamma$ consisting of all the vertices and edges appearing among the simple cycles in any subset of $D$ belonging to the family $F_{d-1}$ is a connected regular graph of degree $d-1$.

In addition to this, the conditions listed below are satisfied with respect to each vertex in $\Gamma$. Let $V$ be any vertex in $\Gamma$ with edges $e_{1}$, $\ldots, e_{d}$ incident at it. Then:

(iv) $V$ is the only vertex which appears among the simple cycle in each of the subsets $D\left\{e_{1}, \ldots, e_{i-1}, e_{i+1}, \ldots, e_{d}\right\}$ for $i=1, \ldots, d$.

(v) $e_{j}$ is the only edge, and the two vertices on $e_{j}$ are the only two vertices which appear among the simple cycles in each of the subsets $D\left\{e_{1}, \ldots, e_{i-1}, e_{i+1}, \ldots, e_{d}\right\}$ for all $i=1, \ldots, d, i \neq j$.

(vi) Let $\left\{e_{j_{1}}, \ldots, e_{j_{r}}\right\}$ be any subset of the edges $e_{1}, \ldots, e_{d}$ with cardinality $r$ between 2 and $d-2$. Then 


$$
\bigcap_{\substack{1 \leqq i \leqq \mathrm{~d} \\ i \notin\left\{j_{1}, \ldots, j_{r}\right\}}} D\left\{e_{1}, \ldots, e_{i-1}, e_{i+1}, \ldots, e_{d}\right\}=D\left\{e_{j_{1}}, \ldots, e_{j_{r}}\right\} .
$$

Proof. To prove the necessity: If $\Gamma$ is the graph of an abstract polytope, let $D$ be the set of all its two-dimensional faces, each of which is a simple cycle in $\Gamma$. If $e_{1}, \ldots, e_{r}$ are edges of $\Gamma$ with a common vertex, then $D\left\{e_{1}, \ldots, e_{r}\right\}$ is the set of two-dimensional faces of the $r$-dimensional face of $\Gamma$ containing $e_{1}, \ldots, e_{r}$ by Theorem 2.6. Hence by Lemmas 2.2 and 2.3, and Theorem 2.6, all the conditions (i) to (vi) are satisfied.

To prove the sufficiency: Let $D$ be a class of simple cycles in $\Gamma$, and $F_{2}, \ldots, F_{d-1}$ the families of subsets of $D$ generated as in 2.12 , which satisfy all the conditions (i) to (vi). For convenience in referring to them, let $f^{1}, f^{2}, \ldots, f^{\mathrm{n}}$ be all the distinct subsets in the family $F_{d-1}$. Associate the symbol $j$ with the subset $f^{j}$ in $F_{d-1}$, for $j=1, \ldots, n$. Let $S=\{1, \ldots, n\}$. Label each vertex of $\Gamma$ by a subset of $S$ by the rule: $j$ is contained in the label on a vertex iff that vertex appears among the simple cycles in $f^{j}$. Let $C$ be the class of all the labels on the vertices of $\Gamma$. We will now prove that $(S, C)$ is an abstract polytope and that $\Gamma$ is its graph.

Axiom 1.1.1 is obviously satisfied by the pair $(S, C)$. Let $V$ be any vertex in $\Gamma$ with edges $e_{1}, \ldots, e_{d}$ incident at it. Then by (iii) each of these subsets, $D\left\{e_{1}, \ldots, e_{i-1}, e_{i+1}, \ldots, e_{d}\right\}$ for $i=1, \ldots, d$, is distinct and these are the only subsets in the family $F_{d-1}$ which contain a simple cycle through $V$. Hence the label on $V$ consists of the symbols associated with these subsets, and hence has cardinality $d$. Also by (iv), $V$ is the only vertex with this label. Hence axiom 1.1.2 is satisfied by the pair $(S, C)$.

Suppose $i$ is the index associated with the subset $D\left\{e_{1}, \ldots, e_{i-1}\right.$, $\left.e_{i+1}, \ldots, e_{d}\right\}$ for $i=1, \ldots, d$. Then the label on $V$ is $\{1, \ldots, d\}$. Let $E$ be any subset of $V$ of cardinality $d-1$, say $E=\{1, \ldots, j-1, j+1, \ldots, d\}$. By (v), the two vertices on edge $e_{j}$ are the only vertices of $\Gamma$ whose labels contain $E$ as a subset. Hence axiom 1.1 .3 is satisfied by the pair $(S, C)$ and a pair of vertices are adjacent on $\Gamma$ if the labels on them contain $d-1$ symbols in common.

Let $V^{*}$ be another vertex in $\Gamma$ such that the intersection of the labels on $V$ and $V^{*}$ is $\{1, \ldots, r\}$ of cardinality $r$ between 1 and $d-2$. Then $V^{*}$ is also contained among the simple cycles in the subset $D e_{1}, \ldots e_{i \ldots 1}$, $\left.e_{i+1}, \ldots, e_{d}\right\}$ for $1 \leqq i \leqq r$. From (vi) this implies that both $V$ and $V^{*}$ are contained among the simple cycles in $D\left\{e_{r+1}, \ldots, e_{d}\right\}$, and the label on 
every vertex appearing among the simple cycles in $D\left\{e_{r+1}, \ldots, e_{d}\right\}$ contains $\{1, \ldots, r\}$ as a subset. By the definition of the subset $D\left\{e_{r+1}\right.$, $\left.\ldots, e_{d}\right\}$, the partial subgraph consisting of the vertices and edges appearing among the simple cycles in it is connected. This implies that there exists an edge path between $V$ and $V^{*}$ such that the label on every vertex along the path contains $\{1, \ldots, r\}$ as a subset. This, and the fact that $\Gamma$ itself is connected, imply that the pair $(S, C)$ satisfies axiom 1.1 .4 also.

Hence $(S, C)$ is an abstract polytope of dimension $d$ with $\Gamma$ as its graph. Condition (vi) implies that $D$ is the class of the two-dimensional faces of this abstract polytope and that each subset in the family $F_{r}$ is the class of two-dimensional faces of some $r$-dimensional face of this abstract polytope.

2.14. Corollary. Given a regular connected graph $\Gamma$, and a class of simple cycles $D$ in it, the necessary and sufficient conditions under which $D$ is the class of two-dimensional faces of an abstract polytope are (i) to (vi) of Theorem 2.13 .

\section{Acknowledgment}

I would like to thank the referee for making numerous suggestions in improving the presentation of this paper.

\section{References}

[1] I. Adler, "Abstract polytopes", Department of Operations Research, Stanford University, Stanford, Calif. (1971).

[2] I. Adler, "Graphs and dual graphs of abstract polytopes", Operations Research Center, University of California, Berkeley, Calif. (September 1971).

[3] I. Adler, G.B. Dantzig and K. G. Murty, "Existence of $x$-paths in abstract poly topes",Technical Report No. 70-1, Department of Operations Research, Stanford University, Stanford, Calif. (January, 1970).

[4] S.S. Cairns, Introductory topology (Ronald Press, New York, 1961).

[5] B. Grünbaum, Convex polytopes (Interscience, New York, 1967).

[6] K.G. Murty, "The generalization of Euler property to abstract polytopes of dimension 3", Department of Industrial Engineering, University of Michigan, Revised 1971. 